Check for updates

Cite this: RSC Adv., 2019, 9, 29522

Received 13th June 2019

Accepted 11th September 2019

DOI: $10.1039 / c 9 r a 04458 b$

rsc.li/rsc-advances

\section{Serum metabolomic alterations in multiple myeloma revealed by targeted and untargeted metabolomics approaches: a pilot study $\uparrow$}

\author{
Venkatesh Chanukuppa, ${ }^{\text {ab }}$ Tushar H. More, ${ }^{\text {ab }}$ Khushman Taunk, (D) a Ravindra Taware, ${ }^{a}$ \\ Tathagata Chatterjee, ${ }^{c}$ Sanjeevan Sharma ${ }^{d}$ and Srikanth Rapole (D) *a
}

\begin{abstract}
Multiple myeloma (MM) is the second most prevalent hematological malignancy characterized by rapid proliferation of plasma cells, which leads to overproduction of antibodies. MM affects around $15 \%$ of all hemato-oncology cases across the world. The present study involves identification of metabolomic alterations in the serum of an MM cohort compared to healthy controls using both LC-MRM/MS based targeted and GC-MS based untargeted approaches. Several MM specific serum metabolomic signatures were observed in this study. A total of 54 metabolites were identified as being significantly altered in MM cohort, out of which, 26 metabolites were identified from LC-MRM/MS based targeted analysis, whereas 28 metabolites were identified from the GC-MS based untargeted analysis. Receiver operating characteristic (ROC) curve analysis demonstrated that six metabolites each from both the datasets can be projected as marker metabolites to discriminate MM subjects with higher specificity and sensitivity. Moreover, pathway analysis deciphered that several metabolic pathways were altered in MM including pyrimidine metabolism, purine metabolism, amino acid metabolism, nitrogen metabolism, sulfur metabolism, and the citrate cycle. Comprehensively, this study contributes valuable information regarding $M M$ induced serum metabolite alterations and their pathways, which could offer further insights into this cancer.
\end{abstract}

\section{Introduction}

Multiple myeloma (MM) is reported as the second most prevalent hematological malignancy $(\sim 15 \%)$ across the world. ${ }^{\mathbf{1}} \mathrm{MM}$ is characterized by rapid proliferation of plasma cells and their subsequent accumulation in bone marrow which results in overproduction of antibodies and eventually leads to bone resorption. ${ }^{2}$ It is one of the most dominant hematological malignancies on the Indian subcontinent, where 4 out of every 100000 people are detected with this cancer every year. ${ }^{3}$ Development of MM is a complex process that is associated with cytological abnormalities such as hyperdiploidy and chromosomal translocations. ${ }^{4}$ Being a heterogeneous disease, MM can be broadly categorized into the following types: monoclonal gammopathy of undetermined clinical significance (MGUS), a benign form of $\mathrm{MM}$; an intermediate stage called as smoldering myeloma and the symptomatic myeloma. ${ }^{5}$ MGUS could

${ }^{a}$ Proteomics Lab, National Centre for Cell Science, Ganeshkhind, Pune-411007, MH, India. E-mail: rsrikanth@nccs.res.in; Fax: +91-20-2569-2259; Tel: +91-20-2570-8075 ${ }^{b}$ Savitribai Phule Pune University, Ganeshkhind, Pune-411007, MH, India

${ }^{c}$ Army Hospital (R\&R), Dhaula Kuan, New Delhi-110010, DL, India

${ }^{d}$ Armed Forces Medical College, Pune-411001, MH, India

$\dagger$ Electronic supplementary information (ESI) available. See DOI: 10.1039/c9ra04458b progress into smoldering myeloma, which finally turns into the symptomatic myeloma. Despite recent developments in the field of hematological malignancy research, there is a significant gap regarding the exact nature of molecular changes that occur during malignant evolution leading to MM.

It is well-known fact that malignant transformations are associated with altered metabolic pathways mostly associated with biosynthetic and bioenergetic processes. ${ }^{6}$ Such metabolic rearrangement or switching depicts an adjustment to aid cell survival, tissue remodeling, tumor growth, as well as cancer metastasis. Strikingly, as evident from available literature, suggests that this metabolic adjustment is coordinated by genomic programming and inveigled by the tumor microenvironment. Moreover, in some situations, the alteration in metabolism plays a crucial role in oncogenesis. ${ }^{6,7}$ Therefore, it is of utmost importance to extract such metabolic deviations by metabolomics approach and understand them in the context of prognosis, therapy response as well as disease pathophysiology.

Metabolomics not only includes the utilization of various analytical methodologies towards identification and quantification of the metabolites pertaining to a biological system, but can monitor the changes at metabolites level in a variety of clinical specimens such as serum, cells, tissue, urine and other biological fluids. ${ }^{\mathbf{8}-17}$ Earlier metabolomics studies in MM were mostly carried out on in vitro models, such as cell lines, to 
investigate metabolic alterations induced by the drug resistance. ${ }^{\mathbf{1 8 , 1 9}}$ Apart from this, identification of potential targets related to apoptosis ${ }^{\mathbf{2 0}}$ was also studied in context of MM. Moreover, few clinical studies were also conducted to investigate the serum metabolomics alterations upon disease remission. ${ }^{21}$ However, to our knowledge, there is no report of comprehensive serum metabolomic investigation using targeted and untargeted metabolomic approaches for $\mathrm{MM}$ to decipher the metabolic alterations crucial for understanding the disease pathophysiology, which in future could be translated into disease diagnosis.

Therefore, in the present study, we have carried out an elaborate MM serum metabolic profiling of human patients compared to healthy control subjects using both targeted and untargeted approaches to identify metabolic fingerprint associated with this disease.

\section{Materials and methods}

\subsection{Subject selection, sample collection and processing}

The study cohort for targeted metabolomic analysis comprised of 48 volunteers $(\mathrm{MM}=24$, control $=24)$ recruited at the Armed Forces Medical College (AFMC), Pune. For untargeted metabolomics analysis, 40 subjects $(\mathrm{MM}=21$, control $=19)$ from the same cohort were utilized. As we collected the samples in a long duration of sample collection period because of the reason that the availability of the samples was scarce for this disease, the number of samples for the LC-MS and GC-MS are different. As we performed the GC-MS analysis earlier, the sample cohort for this analysis has 21 healthy control and $19 \mathrm{MM}$ serum samples. Whereas, later we collected some additional samples, which made the total of 24 serum samples each for healthy controls and MM for the LC-MS analysis. The inclusion criteria for MM cohort consisted of only freshly diagnosed patients with minimum 18 years of age and without any past or present anticancer drug interventions. For the control cohort, age and gender matched individuals devoid of hypertension or diabetes was selected. ESI Table S1† summarizes the demographic information of the study cohort used in this study. The study cohort was advised overnight fasting and blood samples were collected the following morning. $5 \mathrm{~mL}$ of peripheral blood was collected in serum separator tubes (BD Vacutainer ${ }^{\mathrm{TM}} \mathrm{SST}^{\mathrm{TM}}$ II Advance Tubes). The peripheral blood samples were kept for clotting at room temperature and serum separation from the blood was achieved by centrifuging samples at $1500 \times g$ for $5 \mathrm{~min}$ at $15{ }^{\circ} \mathrm{C}$. Serum samples were then transferred to cryo-vials, labeled and subsequently liquid nitrogen were used to snap freeze the samples. Post snap freezing, the serum samples were stored at $-80{ }^{\circ} \mathrm{C}$ ultra-low temperature freezer until further use. All experiments were performed in compliance with guidelines mentioned in Declaration of Helsinki (DoH, 2013). Ethical committees of National Centre for Cell Science (NCCS), Pune and Armed Forces Medical College (AFMC), Pune approved this study (IRB\# NCCS/IEC/2016-I/8). The experimental aspects and investigations to be undertaken were clearly explained to each participants and written informed consent was obtained from them. Each subject participated voluntarily for this study.

\subsection{Targeted LC-MRM/MS based metabolomic profiling}

In-house built MRM library of 120 metabolites was used to investigate the metabolic alterations in the serum of $\mathrm{MM}$ study cohort. These metabolites were selected based on the previous literature survey of various cancer metabolomic studies. $^{22}$ The chromatography parameters and details of MRM library are discussed in our previous report ${ }^{23}$ and the same have been utilized for this study as well. Metabolites extraction was executed by the addition of $200 \mu \mathrm{L}$ methanol to $25 \mu \mathrm{L}$ of every serum samples, vortexed vigorously and incubated at $-20^{\circ} \mathrm{C}$ overnight. Moreover, d2L-phenylalanine (40 ng) was added to the samples prior to extraction, which served as an internal standard. Post incubation, the samples were vortexed vigorously and subjected to $14000 \times g$ centrifugation at $4{ }^{\circ} \mathrm{C}$ for $10 \mathrm{~min}$. The supernatant was then passed through centrifugal filters (Corning ${ }^{\circledR}$ Costar ${ }^{\circledR}$ Spin-X ${ }^{\circledR}$ ) with $0.22 \mu \mathrm{m}$ nylon membrane. Filtrate obtained was then vacuum dried and reconstituted in $25 \mu \mathrm{L}$ of sample reconstitution buffer (a mixture of $6.5 \mathrm{~mL}$ acetonitrile, $2.5 \mathrm{~mL}$ methanol, $1 \mathrm{~mL}$ water and $0.2 \mathrm{~mL}$ formic acid; all the solvents were LCMS grade) for acquiring the samples in positive ionization mode mass spectrometry. In case of negative ionization mode, the vacuum dried metabolites were reconstituted in 25 $\mu \mathrm{L}$ of ultrapure LC-MS grade water. Subsequently, $10 \mu \mathrm{L}$ of each sample was analyzed using a Shimadzu Prominence HPLC system (Shimadzu Corporation, Japan) connected to a triple quadrupole mass spectrometer, 4000 QTRAP (AB SCIEX, USA). For positive mode ionization, metabolites were separated through the XBridge ${ }^{\text {TM }}$ HILIC column $(5 \mu \mathrm{m}, 4.6 \times$ $150 \mathrm{~mm}$, Waters Corp, USA) and Atlantis ${ }^{\mathrm{TM}} \mathrm{T} 3$ column $(5 \mu \mathrm{m}$, $4.6 \times 150 \mathrm{~mm}$, Waters Corp, USA) was used for negative ionization mode. QC samples, prepared from the mixture of pure metabolite standards, were run after every 5 serum metabolite sample injections to assess the uniform instrument performance.

The LC buffers and chromatography parameters along with MS sample acquisition parameters were adopted from our earlier studies as mentioned elsewhere. ${ }^{23}$ Positive ionization chromatographic separation was carried out using $32 \mathrm{~min}$ gradient program of mobile phase A (10 $\mathrm{mM}$ ammonium formate with $0.1 \%$ formic acid in water) and mobile phase $\mathrm{B}$ (acetonitrile with $0.1 \%$ formic acid) with $700 \mu \mathrm{L} \mathrm{min}{ }^{-1}$ elution rate. The gradient started with $95 \% \mathrm{~B}$ and further decreased to $40 \%$ B over next $15 \mathrm{~min}$ and held constant for $6 \mathrm{~min}$, and then the column was gradually returned to $95 \% \mathrm{~B}$ and equilibrated for another $9 \mathrm{~min}$. The negative mode ionization chromatography was carried out with elution rate of $500 \mu \mathrm{L} \mathrm{min}{ }^{-1}$ and 40 min gradient program consisting of $0 \% \mathrm{~B}$ at initial stage and gradually increased to $98 \%$ over next 24 min and maintained for $5 \mathrm{~min}$ in same state and eventually returned to $0 \% \mathrm{~B}$. Finally, the column was equilibrated with $100 \%$ solvent A for 9 min. Mass spectra were acquired using following set of parameters; MS source temperature $400{ }^{\circ} \mathrm{C}$ with interface heater on, curtain gas: 30 and entrance and exit potentials: 10 , two ion source gasses at 45 arbitrary units. LC-MRM/MS data analysis was performed using Analyst 1.5 software (Sciex, USA). 


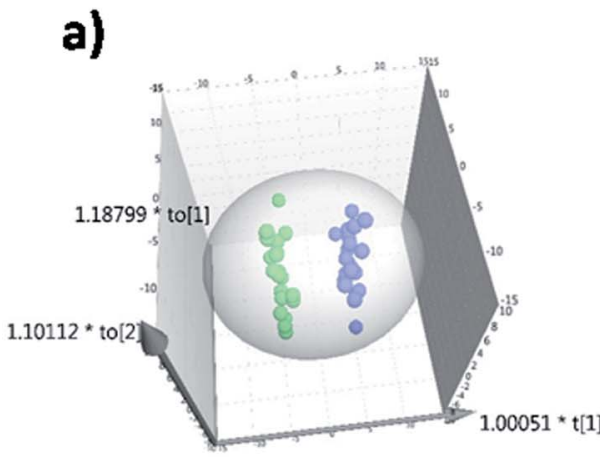

$R 2 \times[1]=0.253 \quad R 2 \times 0[1]=0.157 \quad R 2 \times 0[2]=0.0956$

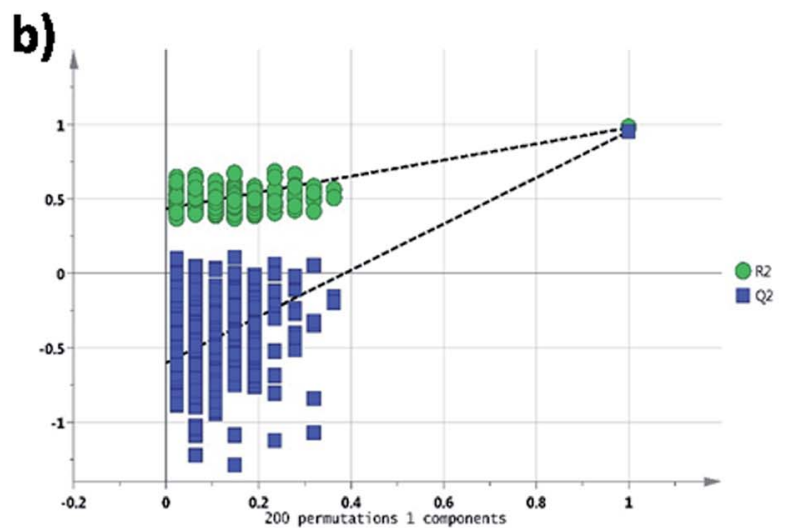

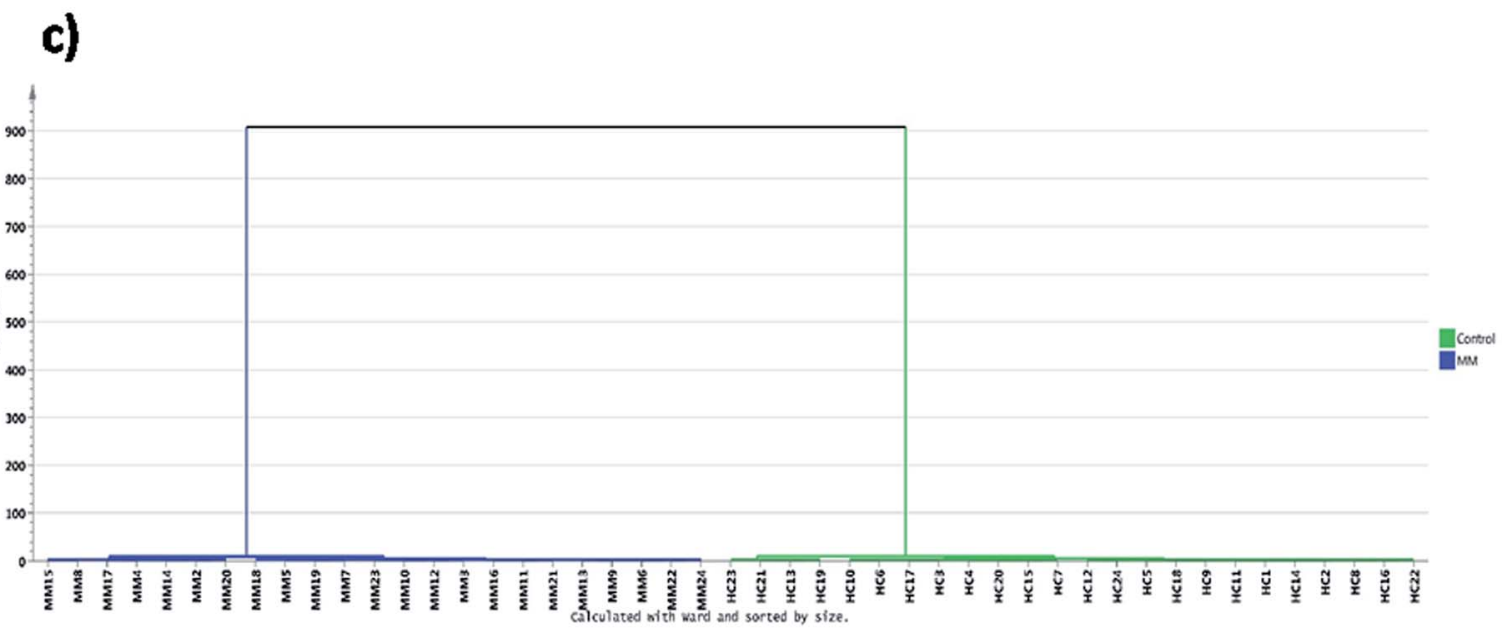

Fig. 1 Multivariate statistical analysis of targeted LC-MRM/MS MM serum metabolomics: (a) distribution of MM subjects ( $n=24$, blue) and healthy controls ( $n=24$, green) in OPLS-DA score plot, (b) permutation validation of OPLS-DA score plot. Total 200 permutations were carried out on OPLS-DA model and obtained $R^{2}=0.978$ and $Q^{2}=0.952$, where $R^{2}$ and $Q^{2}$ indicate the explained variance and predictive ability respectively, (c) hierarchical clustering analysis depicting the segregation of $M M$ (blue) and healthy controls (green).

Analyst quantitation wizard was used for the peak area integration. For elimination of biasness and to maintain quality control measures, samples were randomized during sample analysis and peak integration performed in a blinded manner. Further, the peak area values obtained after performing the integration analysis were exported to a spreadsheet file for further analysis.

\subsection{Untargeted GC-MS-based metabolic profiling}

Metabolite extraction was performed as described in LC-MS methodology. Additionally, two-step derivatization method was applied to the dried metabolite extract to produce methyl and silyl derivatives. First step was methylation, which was achieved by addition of $30 \mu \mathrm{L}$ of methoxyamine hydrochloride solution dissolved in pyridine $\left(20 \mathrm{mg} \mathrm{mL}^{-1}\right)$ and incubating at $37^{\circ} \mathrm{C}$ for $90 \mathrm{~min}$. In the second step, silylation reaction was performed by adding 30 $\mu \mathrm{L}$ of BSTFA with $1 \%$ TMCS solution followed by $1 \mathrm{~h}$ incubation at $70{ }^{\circ} \mathrm{C} .1 \mu \mathrm{L}$ of the derivatized sample was injected into the injection port $\left(250^{\circ} \mathrm{C}\right.$ ) of the Agilent $5975 \mathrm{C} \mathrm{GC}$ system (Agilent Technologies, USA) operating under splitless mode and connected to Agilent 5977A mass selective detector. Metabolites were separated on HP-5
MS ultra-inert fused silica capillary column $(30 \mathrm{~m} \times 0.25 \mu \mathrm{m} \times 0.25$ $\mathrm{mm}$, Agilent, USA). UHP grade helium was used as the carrier gas with a flow rate of $1 \mathrm{~mL} \mathrm{~min}{ }^{-1}$. Following parameters were used to obtain the mass spectra; column inlet temperature $280{ }^{\circ} \mathrm{C}$, MS quadrupole temperature $150^{\circ} \mathrm{C}$, ion source temperature $230^{\circ} \mathrm{C}$. GC oven temperature settings are as follows; the gas chromatography program started with initial oven temperature of $50{ }^{\circ} \mathrm{C}$ held for $2 \mathrm{~min}$, then temperature was gradually increased to $100{ }^{\circ} \mathrm{C}$ at $3^{\circ} \mathrm{C} \mathrm{min}^{-1}$ rate and held for $2 \mathrm{~min}$ and the ramped up further to $200^{\circ} \mathrm{C}$ at $3^{\circ} \mathrm{C} \mathrm{min}^{-1}$ and maintained for $2 \mathrm{~min}$ at this temperature. The column temperature was lowered to $50{ }^{\circ} \mathrm{C}$ in next $5 \mathrm{~min}$ and equilibrated for $2 \mathrm{~min}$. Full scan mode range from $\mathrm{m} / \mathrm{z} 50$ to 550 was used for the metabolite data acquisition. Integration and deconvolution of chromatograms were carried out by using the AMDIS algorithm of Agilent Chemstation software. Metabolites identity was confirmed using NIST 11 standard mass spectral database (NIST, Gaithersburg, MD). Peak areas of the metabolites were exported in a matrix format to spreadsheet file after chromatographic integration. Metabolic standards initiative (MSI) guidelines were followed for performing the all metabolomic experiments. $^{24}$ 
Table 1 Statistically significant differentially regulated serum metabolites identified through the LC-MS based targeted approach

\begin{tabular}{|c|c|c|c|c|c|c|c|c|}
\hline Sr. no. & Metabolite & VIP score & $p$-Value & FDR adjusted $p$-value & Fold change & AUC & $\begin{array}{l}\text { Control } \\
\text { CV\% }\end{array}$ & MM CV\% \\
\hline 1 & NAD & 1.69 & $1.28 \times 10^{-23}$ & $7.97 \times 10^{-22}$ & 17.56 & 0.97 & 16.49 & 13.84 \\
\hline 2 & Adenosine & 1.69 & $1.99 \times 10^{-23}$ & $7.97 \times 10^{-22}$ & 13.78 & 0.97 & 23.65 & 18.12 \\
\hline 3 & CYTIDINE & 1.63 & $1.78 \times 10^{-16}$ & $3.56 \times 10^{-15}$ & 5.56 & 0.97 & 23.68 & 23.49 \\
\hline 4 & Adenine & 1.60 & $9.36 \times 10^{-17}$ & $2.50 \times 10^{-15}$ & 3.62 & 0.98 & 27.44 & 17.32 \\
\hline 5 & Oxaloacetic acid & 1.55 & $9.02 \times 10^{-16}$ & $1.44 \times 10^{-14}$ & 0.01 & 0.99 & 18.23 & 17.46 \\
\hline 6 & Guanosine & 1.51 & $5.17 \times 10^{-14}$ & $6.89 \times 10^{-13}$ & 3.03 & 0.97 & 21.45 & 25.68 \\
\hline 7 & L-Methionine & 1.38 & $2.41 \times 10^{-9}$ & $2.75 \times 10^{-8}$ & 0.60 & 0.94 & 24.58 & 27.84 \\
\hline 8 & Mono-ethylmalonate & 1.38 & $3.06 \times 10^{-9}$ & $3.06 \times 10^{-8}$ & 0.63 & 0.92 & 27.96 & 21.52 \\
\hline 9 & L-Leucine & 1.34 & $4.65 \times 10^{-8}$ & $4.13 \times 10^{-7}$ & 0.67 & 0.91 & 14.94 & 16.75 \\
\hline 10 & L-Homoserine & 1.31 & $2.56 \times 10^{-7}$ & $2.05 \times 10^{-6}$ & 0.64 & 0.90 & 22.34 & 25.73 \\
\hline 11 & L-Threonine & 1.30 & $4.42 \times 10^{-7}$ & $2.95 \times 10^{-6}$ & 0.64 & 0.89 & 25.62 & 21.98 \\
\hline 12 & Thymine & 1.28 & $3.68 \times 10^{-6}$ & $1.92 \times 10^{-5}$ & 0.62 & 0.87 & 18.82 & 16.72 \\
\hline 13 & Methyl malonate & 1.18 & $3.48 \times 10^{-7}$ & $2.53 \times 10^{-6}$ & 1.63 & 0.90 & 12.57 & 15.64 \\
\hline 14 & L-Citrulline & 1.17 & $3.02 \times 10^{-6}$ & $1.86 \times 10^{-5}$ & 0.52 & 0.86 & 18.93 & 21.88 \\
\hline 15 & Uracil & 1.17 & $2.49 \times 10^{-5}$ & $9.04 \times 10^{-5}$ & 0.65 & 0.81 & 28.72 & 24.42 \\
\hline 16 & L-Cysteine & 1.17 & $1.79 \times 10^{-5}$ & $6.83 \times 10^{-5}$ & 0.71 & 0.84 & 22.26 & 26.84 \\
\hline 17 & L-Arginine & 1.15 & $1.79 \times 10^{-5}$ & $6.83 \times 10^{-5}$ & 0.65 & 0.82 & 15.82 & 19.68 \\
\hline 18 & Xanthosine dihydrate & 1.09 & 0.000619 & 0.001415 & 0.62 & 0.76 & 25.68 & 18.94 \\
\hline 19 & Riboflavin & 1.09 & $4.14 \times 10^{-6}$ & $1.95 \times 10^{-5}$ & 2.90 & 0.87 & 19.84 & 23.46 \\
\hline 20 & UTP & 1.08 & $4.98 \times 10^{-5}$ & 0.000159 & 0.49 & 0.92 & 22.56 & 26.78 \\
\hline 21 & Succinic acid & 1.07 & $4.09 \times 10^{-5}$ & 0.000136 & 1.50 & 0.84 & 14.98 & 21.36 \\
\hline 22 & L-Glutamine & 1.06 & $6.66 \times 10^{-5}$ & 0.000197 & 0.67 & 0.81 & 18.96 & 19.14 \\
\hline 23 & Xylitol & 1.04 & 0.000213 & 0.000567 & 1.73 & 0.86 & 22.46 & 21.58 \\
\hline 24 & Adonitol & 1.04 & 0.000124 & 0.000355 & 1.81 & 0.87 & 18.92 & 27.65 \\
\hline 25 & Cytosine & 1.01 & $6.42 \times 10^{-5}$ & 0.000197 & 2.96 & 0.85 & 19.74 & 23.74 \\
\hline 26 & Thyroxine & 1.00 & 0.000147 & 0.000406 & 0.60 & 0.79 & 29.76 & 19.52 \\
\hline
\end{tabular}

\subsection{Data pre-processing and statistical analysis}

The peak areas of identified metabolites in both targeted and untargeted approaches were subjected to statistical analysis using Metaboanalyst 4.0 and SIMCA 14 (Umetrics, Umea, Sweden). Metabolites having missing value $>50 \%$ were discarded from the datasets and those metabolites having $<50 \%$ missing value were subjected to missing value imputation by half of the minimum positive value. Both datasets were normalized to remove the major differences and make metabolic features more comparable to each other. ${ }^{22}$ The LC-MRM/ MS data-set was normalized to median, cube root transformed and range scaling method was used for data scaling. Similarly, GC-MS data was normalized to sum, cube root transformed and auto-scaled. ${ }^{25}$ Univariate and multivariate statistical analyses were applied to these normalized datasets in order to identify the differentially expressed metabolites that can unambiguously discriminate MM subjects from their respective controls. ${ }^{23,26}$ Univariate statistical methods such as Wilcoxon rank $t$-test $(p<0.05)$ and $\log 2$ fold change $(\geq 0.58 /$ $\leq-0.58$ ) were used to select statistically significant metabolites in both the datasets. Furthermore, multivariate statistical methods like principal component analysis (PCA), partial least squares discriminant analysis (PLS-DA) and orthogonal partial least squares discriminant analysis (OPLS-DA) were employed to visualize the overall distribution of serum metabolites among the study cohort as well as their efficacy in discrimination of $\mathrm{MM}$ subjects from the respective controls. Unlike
PCA, which is an unsupervised multivariate statistical approach, PLS-DA and OPLS-DA are supervised multivariate statistical methods and a cross validation is needed to avoid over-fitting of the data. The OPLS-DA model was verified by building 200 random permutation models and comparing their performance $\left(R^{2}\right.$-goodness of fit and $Q^{2}$-predictive ability of the model) with the original model. ${ }^{23}$ Similarly, PLSDA model was also verified. Furthermore, variable importance in projection (VIP) score of OPLS-DA model was used to rank the metabolites as per their contribution in segregation of $\mathrm{MM}$ subjects from the controls in the score plot. To select the marker metabolites, receiver operating characteristic (ROC) curve analysis was carried out to identify the features with highest specificity, sensitivity and accuracy to segregate the MM subjects from controls.

\subsection{Pathway analysis}

Both the datasets, targeted and untargeted were explored towards metabolic pathway analysis for identification of the MM inducible metabolic pathway alterations. The analyses were carried out by MetPA tool of the MetaboAnalyst 3.0 web application (http://metpa.metabolomics.ca). Pathway impact score was used as a measure to identify the altered metabolic pathways. The pathway impact score was calculated based on number of matched metabolites from datasets to a particular metabolic pathway. The hypergeometric test was used for overrepresentation analysis that tested for its enrichment in 


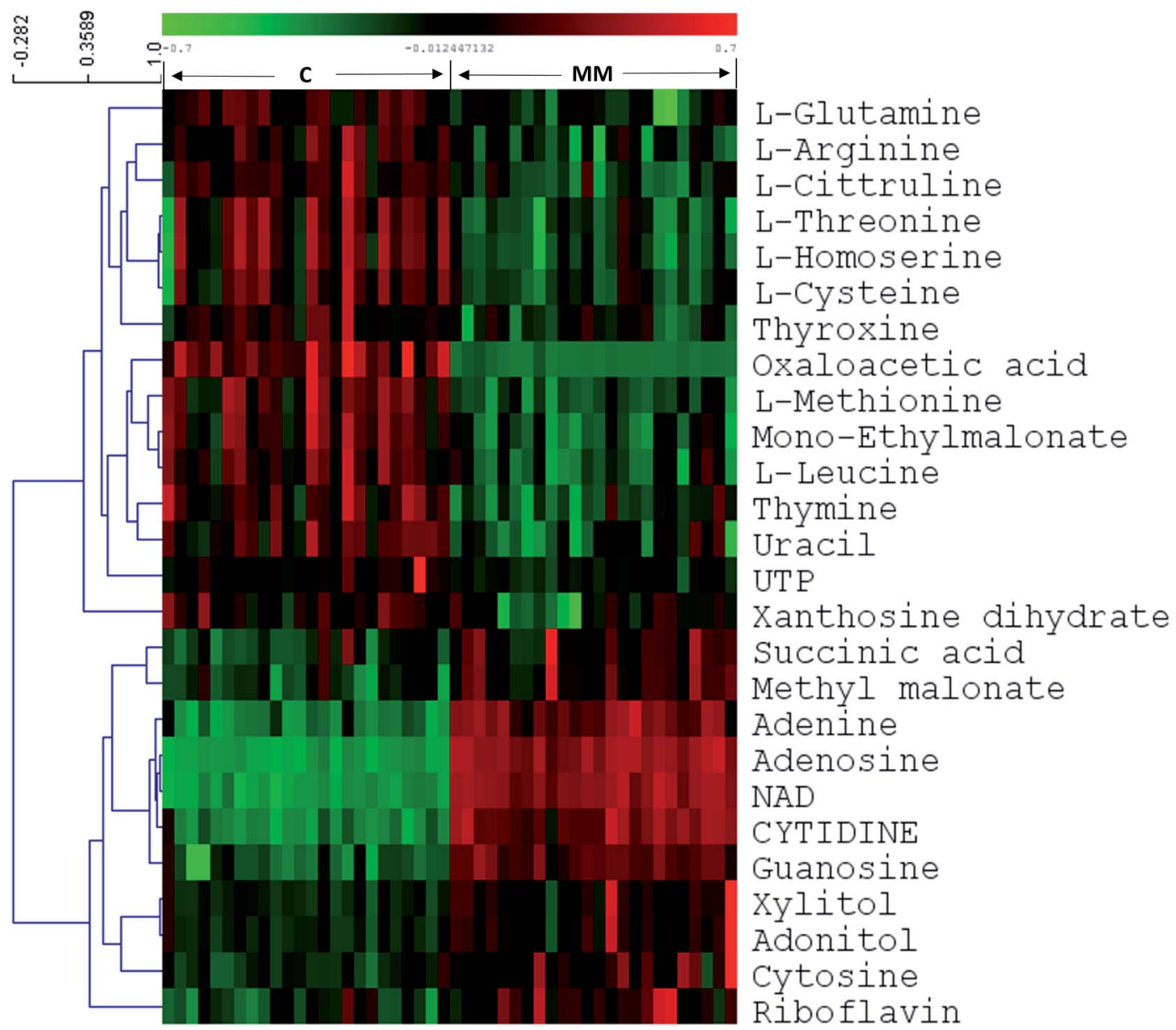

Fig. 2 Heat map of LC-MRM/MS based differential metabolites between healthy controls and MM serum samples. The colors from green to red indicate the increased amount of metabolites (C1-C24: healthy controls, M1-M24: multiple myeloma).

a specific pathway and was compared according to random hits. Further the genes associated with the differentially expressed metabolites were identified using Kyoto Encyclopedia of Genes and Genomes (KEGG) database and Human Metabolome Database (HMDB).

\section{Results}

\subsection{LC-MRM/MS based targeted metabolomic profiling}

In targeted approach, 81 metabolites were consistently detected in all samples out of 120 metabolites targeted. We have built the LC-MRM/MS analysis method for this study by finding the MRM transitions for each metabolite using pure standards purchased from Sigma Aldrich. Representative HILIC and T3 column chromatograms of LC-MRM/MS analysis are presented in ESI Fig. S1.† Univariate statistical methods were applied to identify metabolites that are significantly differing in their concentrations among MM subjects and controls. Further, to extract the MM inducible metabolomic signature, multivariate statistical methods such as PCA, PLSDA and OPLS-DA were carried out. Modest segregation was found in the 2D score plot of the PCA indicating the presence of concentration differences among the metabolites of $\mathrm{MM}$ study cohort (ESI Fig. S2 $\uparrow$ ). Moreover, a supervised multivariate statistical approach such as OPLS-DA was used to sharpen the differences between the MM subjects and their respective controls. Robust group segregation was evident from the OPLS-DA score plot which indicates the metabolic alterations exist in the MM study cohort (Fig. 1a). The model based on OPLS-DA statistics was further authenticated by the permutation test utilizing 200 permutations in between the data groups to avoid biasness due to data over fitting (Fig. 1b). The permutation test yielded $R^{2}$ value of 0.978 and $Q^{2}$ value of 0.952 depicting that the original model has performed better than the permutated models and assures of good prediction ability. VIP score was used as criteria to select the metabolites that had highest impact to group separation in score plot of OPLS-DA. Combination of univariate and multivariate statistical approaches (VIP $>1$, FDR adjusted $p<0.05, \log 2$ FC $>$ $0.58 /<-0.58$ ) were used to propose the panel of metabolites that can discriminate MM subjects from the controls (Table 1). It revealed that 26 metabolites were significantly differed among MM study cohort, out of which 11 metabolites were upregulated while 15 metabolites were down-regulated in MM subjects as compared to control (Table 1). Hierarchical 
clustering analysis depicted distinct clusters of $\mathrm{MM}$ and control subjects, which demonstrate metabolic adaptation of MM subjects is different when compare to healthy controls (Fig. 1c). Further heat map for the differentially expressed metabolites found through LC-MS analysis were showed in Fig. 2 and $\mathrm{S} 4 . \dagger$

\subsection{GC-MS based untargeted metabolomic profiling}

In untargeted approach, 153 metabolites were detected in MM study cohort. Retention time and NIST library metabolite matching score information of the altered metabolites identified using untargeted GC-MS approach is mentioned in ESI Table S2 $\uparrow$ as per the MSI guidelines. ${ }^{24,27}$ For untargeted data analysis, the metabolites that had matching score $>80 \%$ with the NIST library search were only considered. ESI Fig. S3† depicts a representative GC-MS spectrum for MM serum metabolites. The same set of statistical treatments (univariate and multivariate) mentioned above were performed for the untargeted dataset in order to identify the metabolites that are altered significantly upon MM induction. Multivariate statistical models like PCA and OPLS-DA were built from the untargeted dataset to detect the metabolic fingerprint unique to MM. PCA 2D score plot depicted the fairly segregated $\mathrm{MM}$ and control population, which indicates the intrinsic concentration differences exist among the study cohort (ESI Fig. S4 $\dagger$ ). Furthermore, distinct group separation was observed in the OPLS-DA score plot and metabolic features contributing most to the segregation were identified by the VIP score $>1$ (Fig. 3a). A permutation test with 200 permutations was performed to validate the performance of the OPLS-DA model that was built for the MM untargeted metabolite dataset. The permutation test indicates the original model $\left(R^{2}\right.$ value $=0.967$ and $Q^{2}$ value $=$ 0.81 ) is better than permutated models and has not over fitted the data (Fig. 3b). Hierarchical cluster analysis was also in accordance with our earlier findings, revealing clear segregation of study cohort into MM subjects and controls (Fig. 3c). Total 28 most significant metabolites were discovered by using

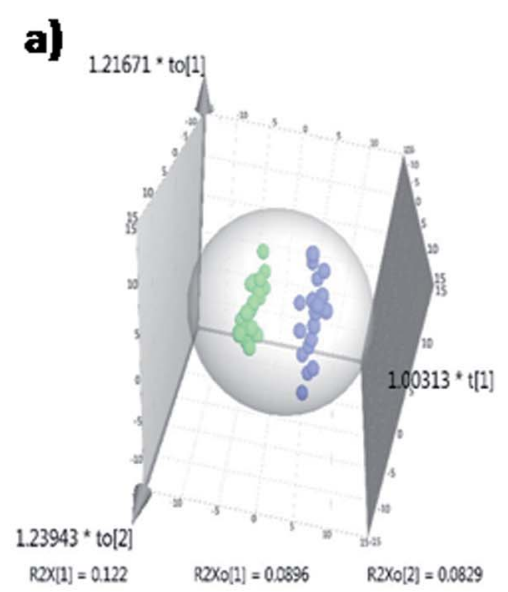

c)

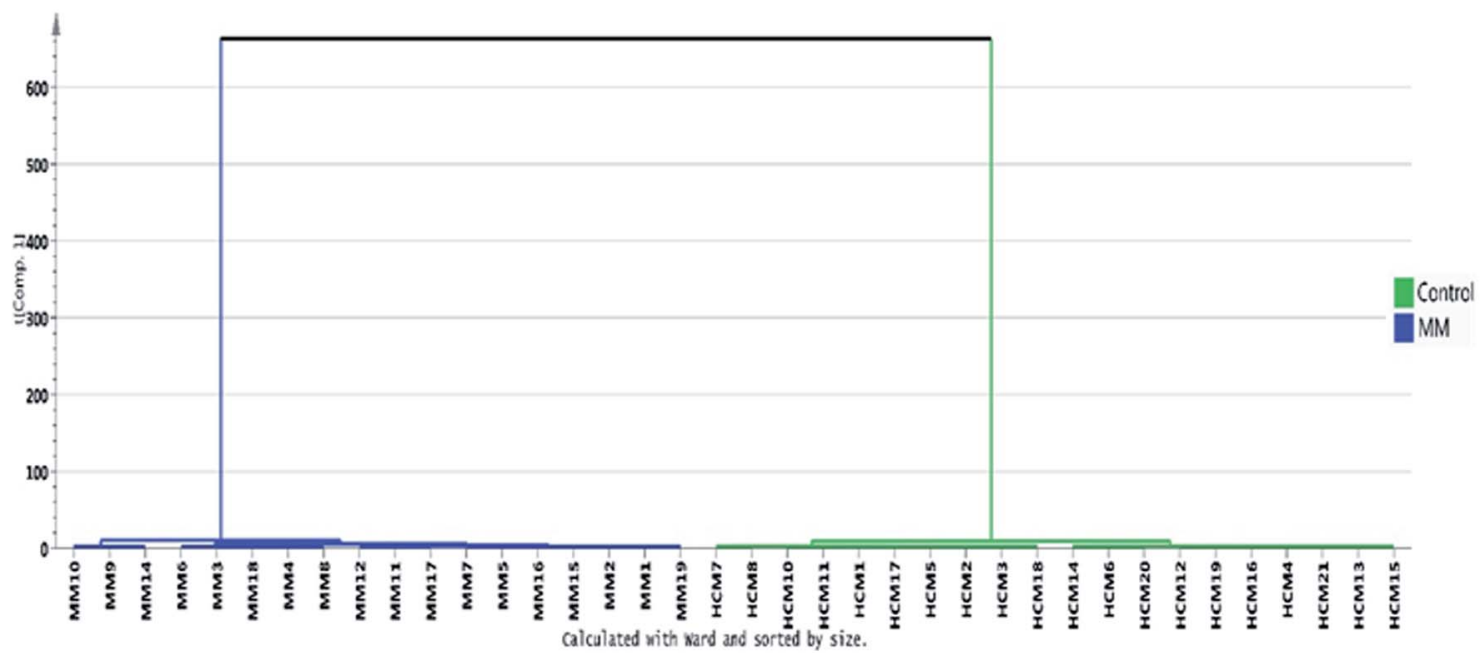

Fig. 3 Multivariate statistical analysis of untargeted GC-MS MM serum metabolomics. (a) OPLS-DA score plot depicting the robust separation of MM subjects ( $n=19$, blue) from healthy controls ( $n=21$, green), (b) permutation validation of OPLS-DA plot obtained after performing 200 random permutations which yielded $R^{2}=0.967$ and $Q^{2}=0.81$, (c) hierarchical clustering analysis representing good clustering of study subjects. 
combination of univariate and multivariate approaches (VIP $>1$, FDR adjusted $p<0.05, \log 2$ FC $>0.58 /<-0.58)$ of which 16 metabolites were up-regulated and 12 metabolites were downregulated in $\mathrm{MM}$ as compared to control (Table 2). Further, heat map for the differentially expressed metabolites found through GC-MS analysis were showed in Fig. 4 and S5. $\dagger$

\subsection{Marker metabolite selection from targeted and untargeted datasets}

Both targeted and untargeted datasets were subjected to the ROC curve analysis to select the metabolites with the highest specificity, sensitivity and accuracy capable of discriminating the MM subjects from respective controls. The area under the curve (AUC) is used as a measure of the predictive ability of a metabolite in ROC curve analysis. Based on AUC, six metabolites each from both the datasets were projected as marker metabolites to discriminate the MM subjects from their respective controls. In targeted LC-MRM/MS dataset, metabolites such as NAD, adenosine, cytidine, adenine, oxaloacetic acid and guanosine were identified as marker metabolites and their expression pattern along with ROC curve plot is represented in Fig. 5a and b. Likewise, in GC-MS analysis, D-ribohexitol, beta-D-glucopyranosiduronic acid, hexadecanoic acid 1,5-anhydro-D-sorbitol, uridine and D-talofuranose are considered as predictive metabolites and their ROC curve plot along with expression pattern is depicted in Fig. $5 c$ and $d$.

\subsection{Identification of altered MM metabolome associated pathways and genes}

To evaluate the impact of MM on metabolic pathways, metabolites from both the MRM based LC-MRM/MS and GC-MS analysis were subjected for pathway analysis using the MetPa tool of MetaboAnalyst 3.0. Pathway analysis results are demonstrated in Fig. 6. Top pathways emerged as pyrimidine metabolism; alanine, aspartate and glutamate metabolism; glycine, serine and threonine metabolism; cysteine and methionine metabolism; purine metabolism; nitrogen metabolism; sulfur metabolism; and citrate cycle (ESI Table S3†). In order to identify the MM differentially regulated metabolites associated genes, 54 serum metabolites were mapped using Kyoto Encyclopedia of Genes and Genomes (KEGG) and human metabolic data base. This analysis resulted in identification of 676 genes which could be related to the MM differentially expressed metabolites (ESI Table S4†).

\section{Discussion}

At present, detection of MM is majorly dependent on morphological criteria and its multi-symptomatic nature makes diagnosis and prognosis challenging. This demands the identification of novel clinically relevant molecular markers and potential targets for developing new therapies for MM. Cancer and metabolism share close connectivity as malignant cells undergoes profound

Table 2 Statistically significant differentially regulated serum metabolites identified through the GC-MS based untargeted approach

\begin{tabular}{|c|c|c|c|c|c|c|c|c|}
\hline Sr. no. & Metabolite & VIP score & $p$-Value & FDR adjusted $p$-value & Fold change & AUC & $\begin{array}{l}\text { Control } \\
\text { CV\% }\end{array}$ & MM CV\% \\
\hline 1 & D-Ribo-hexitol & 2.30 & $4.20 \times 10^{-20}$ & $6.43 \times 10^{-18}$ & 7.08 & 1.00 & 19.36 & 23.58 \\
\hline 2 & Beta-D-glucopyranosiduronic acid & 2.25 & $1.81 \times 10^{-18}$ & $1.39 \times 10^{-16}$ & 5.81 & 1.00 & 28.92 & 23.74 \\
\hline 3 & Hexadecanoic acid & 1.93 & $8.82 \times 10^{-8}$ & $4.50 \times 10^{-6}$ & 1.80 & 0.95 & 18.62 & 14.85 \\
\hline 4 & 1,5-Anhydro-D-sorbitol & 1.84 & $3.49 \times 10^{-7}$ & $1.07 \times 10^{-5}$ & 0.25 & 0.89 & 26.35 & 28.61 \\
\hline 5 & Pseudo uridine & 1.83 & $2.35 \times 10^{-7}$ & $8.97 \times 10^{-6}$ & 6.11 & 0.95 & 12.32 & 16.78 \\
\hline 6 & D-Talofuranose & 1.77 & $1.51 \times 10^{-6}$ & $3.84 \times 10^{-5}$ & 0.37 & 0.91 & 29.84 & 26.74 \\
\hline 7 & Glucofuranoside & 1.66 & $1.54 \times 10^{-5}$ & $3.36 \times 10^{-4}$ & 2.73 & 0.91 & 23.48 & 25.68 \\
\hline 8 & $N$-Acetyl glucosamine & 1.52 & $2.39 \times 10^{-4}$ & $3.03 \times 10^{-3}$ & 0.56 & 0.82 & 19.46 & 23.42 \\
\hline 9 & Purine & 1.51 & $1.15 \times 10^{-4}$ & $1.88 \times 10^{-3}$ & 0.45 & 0.84 & 26.59 & 28.32 \\
\hline 10 & Beta-D-galactopyranoside & 1.51 & $1.23 \times 10^{-4}$ & $1.88 \times 10^{-3}$ & 0.42 & 0.85 & 21.65 & 25.38 \\
\hline 11 & Pyrimidine & 1.50 & $6.16 \times 10^{-5}$ & $1.18 \times 10^{-3}$ & 0.40 & 0.83 & 20.28 & 22.07 \\
\hline 12 & 2-Piperidinecarboxylic acid & 1.50 & $1.60 \times 10^{-4}$ & $2.22 \times 10^{-3}$ & 0.44 & 0.81 & 14.63 & 16.09 \\
\hline 13 & D-Lactose & 1.42 & $3.18 \times 10^{-3}$ & $2.01 \times 10^{-2}$ & 1.64 & 0.75 & 12.59 & 16.83 \\
\hline 14 & D-Xylofuranose & 1.42 & $4.41 \times 10^{-4}$ & $4.49 \times 10^{-3}$ & 2.80 & 0.77 & 15.97 & 16.23 \\
\hline 15 & L-Threonine & 1.42 & $2.06 \times 10^{-3}$ & $1.50 \times 10^{-2}$ & 0.20 & 0.69 & 15.86 & 14.93 \\
\hline 16 & Nonadecanoic acid & 1.41 & $8.80 \times 10^{-4}$ & $8.42 \times 10^{-3}$ & 2.36 & 0.83 & 24.46 & 26.66 \\
\hline 17 & L-Threitol & 1.40 & $3.58 \times 10^{-4}$ & $3.92 \times 10^{-3}$ & 2.24 & 0.81 & 26.32 & 27.39 \\
\hline 18 & D-Glucopyranose & 1.39 & $3.00 \times 10^{-3}$ & $2.00 \times 10^{-2}$ & 2.39 & 0.81 & 22.71 & 25.80 \\
\hline 19 & 2-Alpha-mannobiose & 1.38 & $5.87 \times 10^{-3}$ & $3.10 \times 10^{-2}$ & 1.50 & 0.73 & 27.28 & 29.84 \\
\hline 20 & Cystathionine & 1.33 & $3.51 \times 10^{-3}$ & $2.07 \times 10^{-2}$ & 0.63 & 0.80 & 23.40 & 14.67 \\
\hline 21 & Stearate & 1.27 & $2.82 \times 10^{-3}$ & $1.96 \times 10^{-2}$ & 2.11 & 0.87 & 15.82 & 13.26 \\
\hline 22 & Inositol & 1.25 & $1.42 \times 10^{-3}$ & $1.15 \times 10^{-2}$ & 1.54 & 0.80 & 18.54 & 19.23 \\
\hline 23 & L-Asparagine & 1.20 & $1.94 \times 10^{-3}$ & $1.48 \times 10^{-2}$ & 0.49 & 0.85 & 25.63 & 27.52 \\
\hline 24 & Arachidonic acid & 1.20 & $1.13 \times 10^{-2}$ & $4.93 \times 10^{-2}$ & 1.60 & 0.71 & 16.92 & 20.84 \\
\hline 25 & Beta-D-galactofuranose & 1.19 & $7.39 \times 10^{-3}$ & $3.54 \times 10^{-2}$ & 2.04 & 0.84 & 24.36 & 19.56 \\
\hline 26 & D-Xylopyranose & 1.18 & $1.31 \times 10^{-3}$ & $1.15 \times 10^{-2}$ & 0.23 & 0.79 & 25.87 & 27.32 \\
\hline 27 & Stigmasterol & 1.18 & $1.38 \times 10^{-3}$ & $1.15 \times 10^{-2}$ & 0.54 & 0.80 & 28.39 & 27.65 \\
\hline 28 & Maltitol & 1.10 & $7.65 \times 10^{-3}$ & $3.55 \times 10^{-2}$ & 3.51 & 0.74 & 26.57 & 28.34 \\
\hline
\end{tabular}




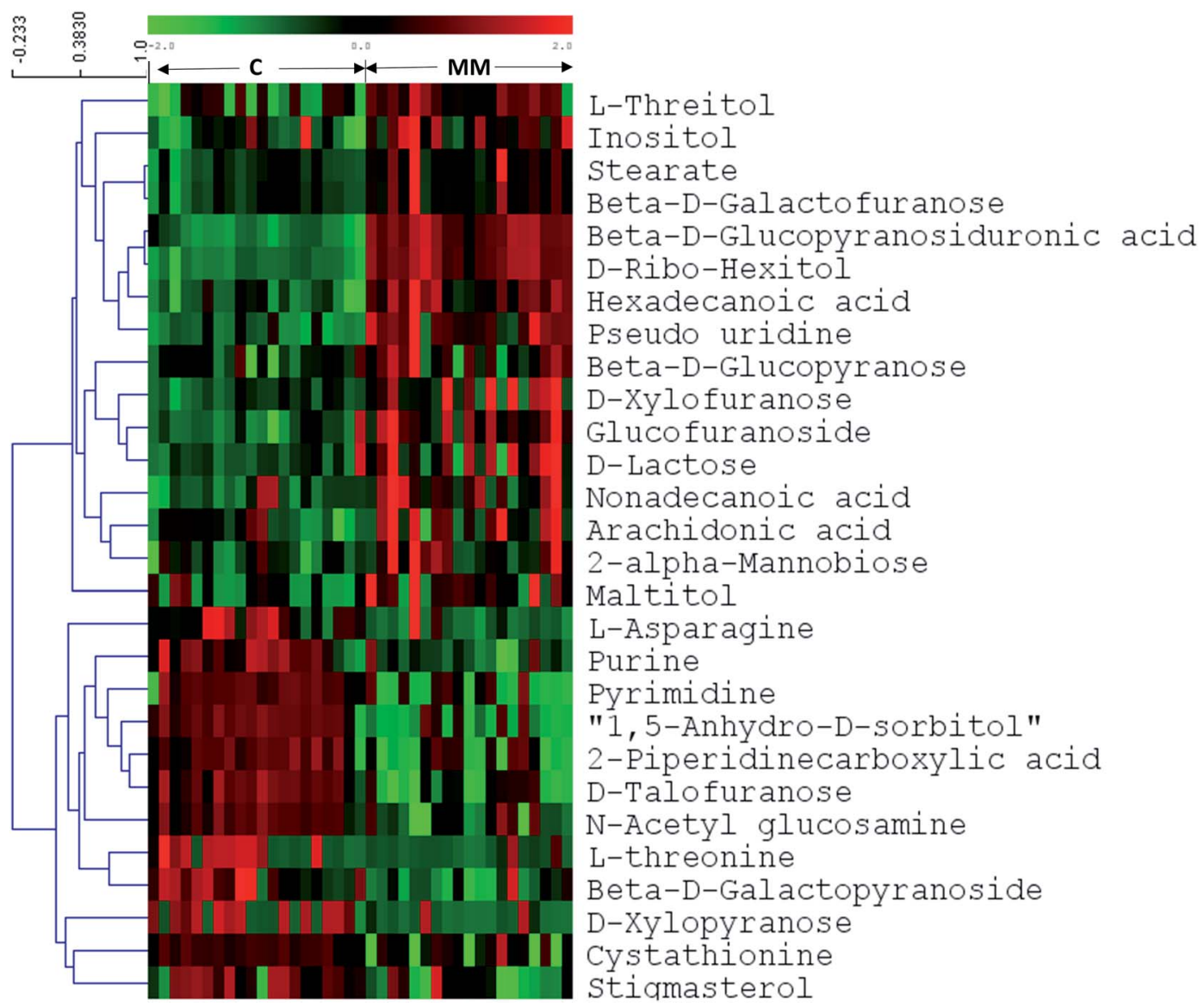

Fig. 4 Heat map of GC-MS based differential metabolites between healthy controls and MM serum samples. The colors from green to red indicate the increased amount of metabolites (C1-C21: healthy controls, M1-M19: multiple myeloma).

metabolic rearrangements to support its proliferative nature and bioenergetics. Hence, in this study, we attempted to explore the serum metabolomic alterations in MM using LC-MRM/MS based targeted as well as GC-MS based untargeted metabolomics. The study showed profound changes in serum metabolites of MM patients as compared to controls. The major metabolic changes identified are discussed further herewith.

Targeted LC-MRM/MS analysis unveiled significant alterations of 26 metabolites in MM serum as compared to control. These differentially expressed metabolites consist of 11 upregulated and 15 down-regulated metabolites. NAD was observed to be up-regulated in MM subjects as compared to the controls, which is considered as a key molecule in various biological processes including energy metabolism and enzymatic regulation. ${ }^{28}$ It is also the substrate for non-redox reactions that affect gene expression such as $\mathrm{Ca}^{2+}$ transport and apoptosis. ${ }^{29} \mathrm{NAD}$ is involved in the important post-translational modifications of proteins such as acylation and deacylation by SIRTs enzymes. ${ }^{30}$ Moreover, the aberrant NAD metabolism is reported in various cancers. ${ }^{31}$ Adenosine, which is found to be increased in MM serum, is an important immunosuppressive molecule. ${ }^{32}$ Extracellular adenosine bind to adenosine receptors of immune cells suppressing the pro-inflammatory activity of the cells. ${ }^{32}$ Furthermore, the activation of adenosine receptors increases inflammatory molecules and immunoregulatory cells governing a long-lasting immunosuppressive environment. ${ }^{32}$ This leads to the discouragement of any antitumor immune responses induced by the body. Oxaloacetic acid (OAA), a key intermediate in the TCA cycle involved in energy production is downregulated in MM subjects when compared with controls. The reason for depleted levels of oxaloacetic acid in serum could be explained by the fact that oxaloacetate along with citrate is used to generate acetyl COA and aspartate that is used in the synthesis of nucleotides and lipids quite extensively in proliferating cells. It is also in agreement with the earlier report that OAA can induce apoptosis in hepatocellular carcinoma cells via inhibition of glycolysis and restoring oxidative phosphorylation. $^{33}$ Thus, decreased levels of OAA in MM serum samples also suggest a prevalence of glycolysis over oxidative phosphorylation in MM subjects. Guanosine is formed as an intermediate of the nucleic acid breakdown. ${ }^{34}$ During purine salvage, guanosine can be cleaved further to release guanine, which is salvaged back to GMP by the enzyme hypoxanthineguanine phosphoribosyl transferase (HGPRT). ${ }^{34}$ Increased concentrations of guanosine in MM serum imply that the purine salvage is highly active in MM. In addition, elevated levels of cytidine, and adenine in MM represents an overall increase in nucleotide metabolism to support proliferation. ${ }^{34}$ 
a)

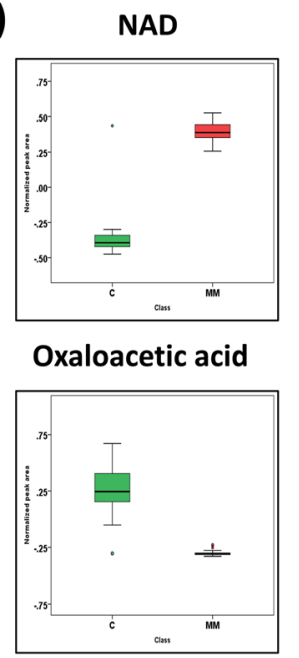

b)

\section{D-Ribo-Hexitol}

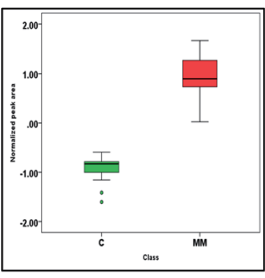

Pseudo uridine

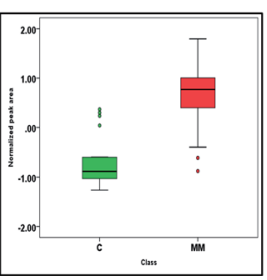

Adenosine

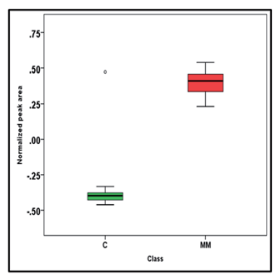

Guanosine

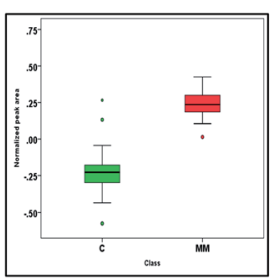

Cytidine
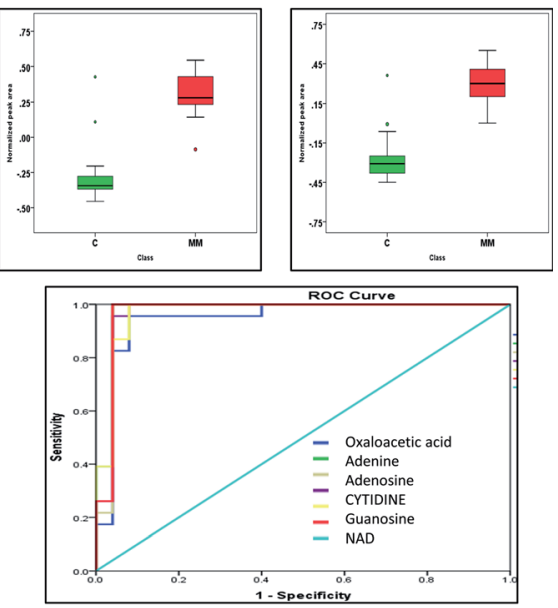

Glucopyrano. a.

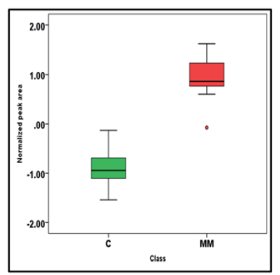

D-Talofuranose

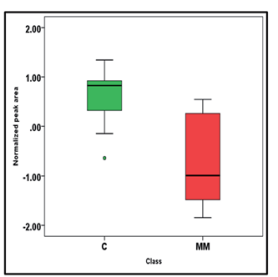

Hexadecanoic acid

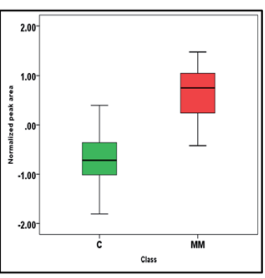

Anhydro-D-sorbitol
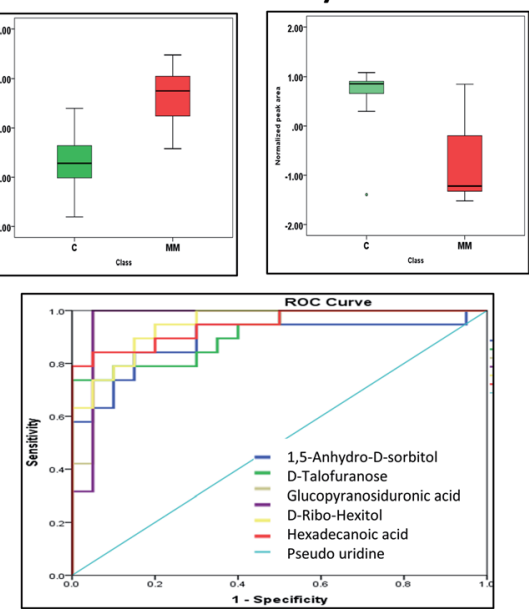

Fig. 5 Marker metabolites selected by ROC curve analysis (a) concentration differences between significant metabolites from targeted analysis of MM (red) and healthy control (green) samples demonstrated by box-and-whisker plots, (b) the ROC curve analysis of marker metabolites viz. NAD (AUC $=$ 0.97, sensitivity $=0.95$, specificity $=0.96, \mathrm{Cl}=95 \%)$, adenosine $(\mathrm{AUC}=0.97$, sensitivity $=0.95$, specificity $=0.92, \mathrm{Cl}=95 \%), \mathrm{cytidine}(\mathrm{AUC}=0.97$, sensitivity $=0.91$, specificity $=0.96, \mathrm{Cl}=95 \%$ ), adenine ( $\mathrm{AUC}=0.98$, sensitivity $=0.95$, specificity $=0.96, \mathrm{Cl}=95 \%)$, oxaloacetic acid $(A \cup C=0.99$, sensitivity $=0.92$, specificity $=0.97, \mathrm{Cl}=95 \%$ ) and guanosine ( $\mathrm{AUC}=0.97$, sensitivity $=0.87$, specificity $=0.88, \mathrm{Cl}=95 \%)$ from targeted analysis, $(\mathrm{c}$ ) concentration differences between significant metabolites from untargeted analysis of MM (red) and healthy control (green) samples demonstrated by box-and-whisker plots, (d) the ROC curve analysis of marker metabolites viz. D-ribohexitol (AUC $=1$, sensitivity $=0.94$, specificity $=0.95, \mathrm{Cl}=95 \%$ ), beta-D-glucopyranosiduronic acid ( $A \cup C=1$, sensitivity $=0.94$, specificity $=0.95, \mathrm{Cl}=95 \%$ ), hexadecanoic acid (AUC $=0.95$, sensitivity $=0.84$, specificity $=0.85, \mathrm{Cl}=95 \%$ ), anhydro-D-sorbitol ( $A \cup C=0.89$, sensitivity $=0.80$, specificity $=0.85, \mathrm{Cl}=95 \%$ ), uridine (AUC $=0.95$, sensitivity $=0.84$, specificity $=0.90, \mathrm{Cl}=95 \%$ ) and $\mathrm{D}$-talofuranose ( $\mathrm{AUC}=0.91$, sensitivity $=0.75$, specificity $=0.79, \mathrm{Cl}=95 \%$ ) from $\mathrm{GC}$ - $\mathrm{MS}$ analysis.

In untargeted GC-MS analysis, significant changes in 28 metabolites were observed that included 16 up-regulated and 12 down-regulated metabolites. Concentration of hexadecanoic acid (palmitic acid) was found to be increased in MM serum as compared to controls. It is reported that increased palmitic acid level is associated with TL4 mediated invasiveness in pancreatic cancer. ${ }^{35}$ Moreover, palmitic acid supplementation has shown to rescue adiponectin-induced apoptosis in MM cells. ${ }^{36}$ Therefore, our observation of increased palmitic acid in MM might be linked with its role in invasiveness and proliferation that needs to be further investigated. Further, decrement in 1,5-anhydro-Dsorbitol was observed in MM serum, which is an indicator of glycaemic control. Its levels manifest the activity of polyol pathways that are mostly functional in hyper glycaemic conditions. ${ }^{37}$ Increase in uridine levels reflects active involvement of pyrimidine metabolism which is common metabolic alteration observed in cancer. ${ }^{34}$ It is well-known fact that, $\mathrm{N}$ or $\mathrm{O}$ linked $\mathrm{N}$ acetylglucosamine (GlcNAc) is one of the prominent posttranslational modifications observed on proteins displayed on the cell surface of malignant cells. Moreover, it is now established that GlcNAc modified proteins aid in cell migration and invasion. ${ }^{38}$ GlcNAc is reported to be mediating cell signaling directly and indirectly. ${ }^{39}$ The modification of epithelial cadherin (Ecadherin) with O-linked $\beta$-GlcNAc is reported to impair cell 
adhesion and promote tumour cell invasion. ${ }^{40}$ We have observed decreased levels of GalNAc in MM serum which strengthens the hypothesis of its preferred utilization for post-translational protein glycosylation in malignant condition. For rapid and continuous synthesis of cell constituting basic machinery, proliferating cancerous cells have high demands of nucleotides. This gets achieved by either nucleotide salvage pathways of purines and pyrimidines or de novo synthesis or a combination of both. ${ }^{34}$ Nucleotide salvage pathway utilizes downstream components of degraded nuclear material like DNA or RNA in the form of purines and pyrimidines to resynthesize the dNTPs for DNA/ RNA formation. ${ }^{34}$ Decrement of both purines and pyrimidines in GC-MS analysis reinforce hyperactivity of the nucleotide salvage pathway to fulfil the proliferative demand.

The significant metabolites obtained from both approaches were further evaluated for their role in various physiological pathways. The metabolic pathway analysis revealed perturbations in alanine, aspartate and glutamate metabolism, pyrimidine metabolism, cysteine and methionine metabolism, glycine, serine and threonine metabolism, purine metabolism, nitrogen metabolism, sulfur metabolism, and citrate cycle (TCA cycle). Several metabolites from purine and pyrimidine metabolism have been deregulated which is in good agreement with previous reports from various cancer metabolomics studies. ${ }^{41}$ Sulfur metabolism is important in the generation of sulfurcontaining amino acids that perform many vital functions. ${ }^{42}$ Alterations in the TCA cycle, as observed in this study, has been demonstrated to play a vital role in carcinogenesis. ${ }^{43}$ Our results demonstrate that certain key metabolic pathways including NAD metabolism, TCA cycle, nucleotide metabolism and sulphur metabolism etc. gets altered in MM and targeting these pathways and its intermediary metabolites may help in developing novel targets for treating MM.

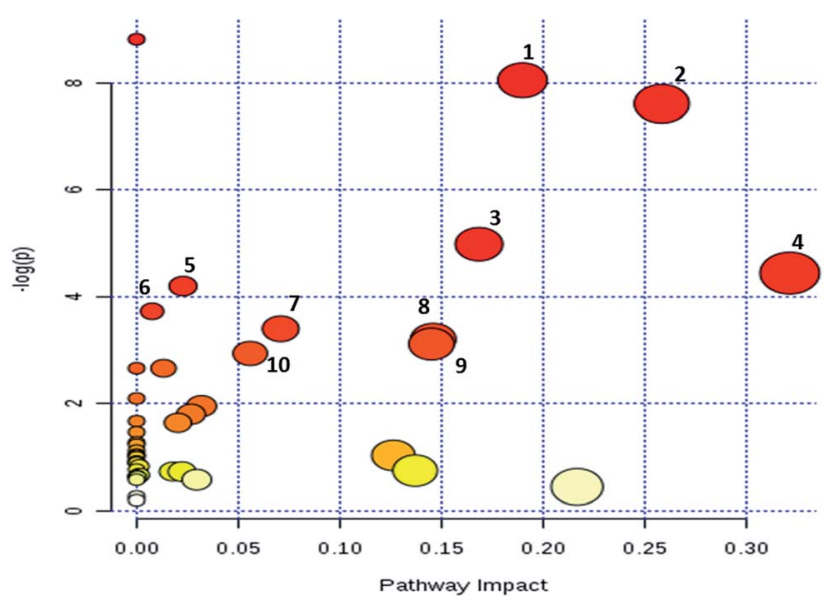

Fig. 6 Differentially regulated metabolic pathway analysis of MM serum metabolites. Metabolic pathway map of differentially regulated metabolites generated using MetPa tool of MetaboAnalyst web application. Significant pathways identified includes (1) pyrimidine metabolism, (2) alanine, aspartate and glutamate metabolism, (3) glycine, serine and threonine metabolism, (4) cysteine and methionine metabolism, (5) purine metabolism, (6) nitrogen metabolism, (7) sulfur metabolism, (8) citrate cycle (TCA cycle), (9) riboflavin metabolism (10) pentose and glucuronate interconversions.

\section{Conclusions}

Overall, the present study demonstrates the potential of serum metabolomics approach towards the segregation of MM cohort from healthy controls. Increased metabolite coverage was obtained with the use of different metabolomics platforms such as LC-MRM/MS and GC-MS. The results obtained are noteworthy and helpful in discrimination of MM individuals from healthy controls. We believe that these results may be useful in future diagnostic, prognostic and therapeutic development. In addition, these results will help to understand the disease pathophysiology of MM in the context of altered cancer metabolism. However, thorough validation of the inferences drawn from this study in a large cohort of samples is required before its implementation in the clinical setting. Validation in large cohort of patients for the metabolite based biosignature, identified in this study, can help diagnose MM in early stages. Early diagnosis will eventually lead to proper on-time therapeutic interventions from the clinicians, which could help in better management of this disease, thereby improving the patient life and survival expectancy.

\section{Author contributions}

Conceived the study: VC, TC, SS, SR; designed the study: VC, TC, SS, SR; performed the experiments: VC, THM; compiled and analyzed data: VC, THM, KT, RT, SR; statistical analysis: VC, THM, SR; provided clinical samples: TC, SS; provided chemicals and reagents: SR. All authors reviewed the manuscript and contributed in writing.

\section{Conflicts of interest}

Authors declare that they do not have any conflict of interests.

\section{Acknowledgements}

General: We thank all the volunteers for participating in this study. Funding: authors would like to acknowledge Department of Biotechnology (DBT), Govt. of India, grant no. BT/PR10855/ $\mathrm{BRB} / 10 / 1330 / 2014$ and NCCS intramural funding. VC and RT acknowledge Council of Scientific and Industrial Research (CSIR), New Delhi, India for fellowship.

\section{References}

1 M. H. Z. Guang, A. McCann, G. Bianchi, L. Zhang, P. Dowling, D. Bazou, P. O'Gorman and K. C. Anderson, Leuk. Lymphoma, 2018, 59, 542-561.

2 T. Hideshima, P. L. Bergsagel, W. M. Kuehl and K. C. Anderson, Blood, 2004, 104, 607-618.

3 O. Landgren and B. Weiss, Leukemia, 2009, 23, 1691.

4 V. Scudla, M. Zemanova, J. Minarik, J. Bacovsky, M. Ordeltova, K. Indrak, M. Budikova, L. Dusek and V. Farbiakova, Neoplasma, 2006, 53, 277-284.

5 W. M. Kuehl and P. L. Bergsagel, Nat. Rev. Cancer, 2002, 2, 175. 
6 M. D. Hirschey, R. J. DeBerardinis, A. M. E. Diehl, J. E. Drew, C. Frezza, M. F. Green, L. W. Jones, Y. H. Ko, A. Le and M. A. Lea, Semin. Cancer Biol., 2015, s129-s150.

7 P. Mishra and S. Ambs, Mol. Cell. Oncol., 2015, 2, e992217.

8 W. B. Dunn, D. Broadhurst, P. Begley, E. Zelena, S. FrancisMcIntyre, N. Anderson, M. Brown, J. D. Knowles, A. Halsall and J. N. Haselden, Nat. Protoc., 2011, 6, 1060.

9 O. Fiehn, J. Kopka, P. Dörmann, T. Altmann, R. N. Trethewey and L. Willmitzer, Nat. Biotechnol., 2000, 18, 1157.

10 E. Zelena, W. B. Dunn, D. Broadhurst, S. Francis-McIntyre, K. M. Carroll, P. Begley, S. O'Hagan, J. D. Knowles, A. Halsall and HUSERMET Consortium, Anal. Chem., 2009, 81, 1357-1364.

11 C. Gieger, L. Geistlinger, E. Altmaier, M. H. De Angelis, F. Kronenberg, T. Meitinger, H.-W. Mewes, H.-E. Wichmann, K. M. Weinberger and J. Adamski, PLoS Genet., 2008, 4, e1000282.

12 R. Pandher, C. Ducruix, S. Eccles and F. Raynaud, J. Chromatogr. B: Anal. Technol. Biomed. Life Sci., 2009, 877, 1352-1358.

13 J. Munger, B. D. Bennett, A. Parikh, X.-J. Feng, J. McArdle, H. A. Rabitz, T. Shenk and J. D. Rabinowitz, Nat. Biotechnol., 2008, 26, 1179.

$14 \mathrm{~W}$. Welthagen, R. A. Shellie, J. Spranger, M. Ristow, R. Zimmermann and O. Fiehn, Metabolomics, 2005, 1, 65-73.

15 M. R. Pears, J. D. Cooper, H. M. Mitchison, R. J. MortishireSmith, D. A. Pearce and J. L. Griffin, J. Biol. Chem., 2005, 280(52), 42508-42514.

16 W. B. Dunn, D. Broadhurst, D. I. Ellis, M. Brown, A. Halsall, S. O'hagan, I. Spasic, A. Tseng and D. B. Kell, Int. J. Epidemiol., 2008, 37, i23-i30.

17 H. G. Gika, G. A. Theodoridis and I. D. Wilson, J. Chromatogr. A, 2008, 1189, 314-322.

18 K. A. Zub, M. M. L. de Sousa, A. Sarno, A. Sharma, A. Demirovic, S. Rao, C. Young, P. A. Aas, I. Ericsson and A. Sundan, PLoS One, 2015, 10, e0119857.

19 D. R. Jones, Z. Wu, D. Chauhan, K. C. Anderson and J. Peng, Anal. Chem., 2014, 86, 3667-3675.

20 C. Bardeleben, S. Sharma, J. R. Reeve, S. Bassilian, P. J. Frost, B. Hoang, Y. Shi and A. Lichtenstein, Mol. Cancer Ther., 2013, 12(7), 1310-1321.

21 L. Puchades-Carrasco, R. Lecumberri, J. Martínez-López, J. J. Lahuerta, M. V. Mateos, F. Prósper, J. San Miguel and A. Pineda-Lucena, Clin. Cancer Res., 2013, 19(17), 4770-4779.
22 T. H. More, S. RoyChoudhury, J. Christie, K. Taunk, A. Mane, M. K. Santra, K. Chaudhury and S. Rapole, OncoTargets Ther., 2018, 9, 2678.

23 T. H. More, R. Taware, K. Taunk, V. Chanukuppa, V. Naik, A. Mane and S. Rapole, Metabolomics, 2018, 14, 107.

24 L. W. Sumner, A. Amberg, D. Barrett, M. H. Beale, R. Beger, C. A. Daykin, T. W.-M. Fan, O. Fiehn, R. Goodacre and J. L. Griffin, Metabolomics, 2007, 3, 211-221.

25 J. Xia, I. V. Sinelnikov, B. Han and D. S. Wishart, Nucleic Acids Res., 2015, 43, W251-W257.

26 H. Flach, M. Rosenbaum, M. Duchniewicz, S. Kim, S. L. Zhang, M. D. Cahalan, G. Mittler and R. Grosschedl, Immunity, 2010, 33, 723-735.

27 O. Fiehn, D. Robertson, J. Griffin, M. van der Werf, B. Nikolau, N. Morrison, L. W. Sumner, R. Goodacre, N. W. Hardy and C. Taylor, Metabolomics, 2007, 3, 175-178. 28 A. Nikiforov, V. Kulikova and M. Ziegler, Crit. Rev. Biochem. Mol. Biol., 2015, 50, 284-297.

29 D. Surjana, G. M. Halliday and D. L. Damian, J. Nucleic Acids, 2010, 157591.

30 A. R. Stram and R. M. Payne, Cell. Mol. Life Sci., 2016, 73, 4063-4073.

31 B. Poljsak, J. Clin. Exp. Oncol., 2016, 5, 4.

32 J. Stagg and M. Smyth, Oncogene, 2010, 29, 5346.

33 Y. Kuang, X. Han, M. Xu and Q. Yang, Cancer Med., 2018, 7, 1416-1429.

34 C. K. Mathews, Nat. Rev. Cancer, 2015, 15, 528.

35 M. J. Binker-Cosen, D. Richards, B. Oliver, H. Y. Gaisano, M. G. Binker and L. I. Cosen-Binker, Biochem. Biophys. Res. Commun., 2017, 484, 152-158.

36 E. Medina, K. Oberheu, S. Polusani, V. Ortega, G. Velagaleti and B. Oyajobi, Leukemia, 2014, 28, 2080.

37 A. Ferretti, S. D'Ascenzo, A. Knijn, E. Iorio, V. Dolo, A. Pavan and F. Podo, Br. J. Cancer, 2002, 86, 1180.

38 D. J. Gill, K. M. Tham, J. Chia, S. C. Wang, C. Steentoft, H. Clausen, E. A. Bard-Chapeau and F. A. Bard, Proc. Natl. Acad. Sci. U. S. A., 2013, 110, E3152-E3161.

39 J. B. Konopka, Scientifica, 2012, 489208.

40 Y. R. Yang, D. H. Kim, Y.-K. Seo, D. Park, H.-J. Jang, S. Y. Choi, Y. H. Lee, G. H. Lee, K. Nakajima and N. Taniguchi, OncoTargets Ther., 2015, 6, 12529.

41 A. N. Lane and T. W.-M. Fan, Nucleic Acids Res., 2015, 43, 2466-2485.

42 J. T. Brosnan and M. E. Brosnan, J. Nutr., 2006, 136, 1636S1640S.

43 S. Cardaci and M. R. Ciriolo, Int. J. Cell Biol., 2012, 161837. 